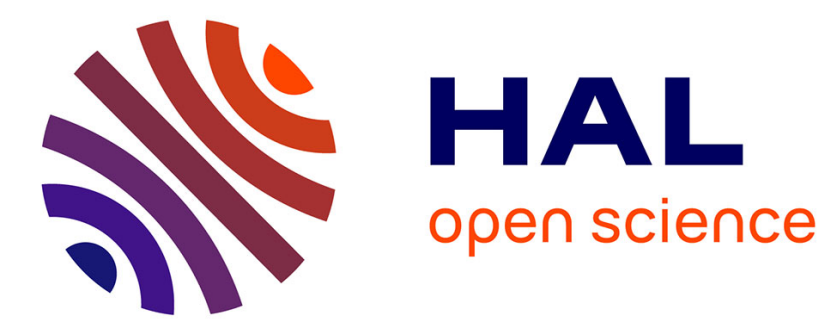

\title{
Initial runs of the NEMO 3 experiment
}

Sophie Jullian

\section{To cite this version:}

Sophie Jullian. Initial runs of the NEMO 3 experiment. Workshop on Neutrino Oscillations and their

Origins (NOON03) 4, Feb 2003, Kanazawa, Japan. pp.291-299. in2p3-00021842

\section{HAL Id: in2p3-00021842 \\ https://hal.in2p3.fr/in2p3-00021842}

Submitted on 24 May 2004

HAL is a multi-disciplinary open access archive for the deposit and dissemination of scientific research documents, whether they are published or not. The documents may come from teaching and research institutions in France or abroad, or from public or private research centers.
L'archive ouverte pluridisciplinaire HAL, est destinée au dépôt et à la diffusion de documents scientifiques de niveau recherche, publiés ou non, émanant des établissements d'enseignement et de recherche français ou étrangers, des laboratoires publics ou privés. 
LAL 03-29

June 2003

INITIAL RUNS OF THE NEMO 3 EXPERIMENT

\author{
NEMO Collaboration \\ CENBG, IN2P3-CNRS et Université de Bordeaux, 33170 Gradignan, France \\ LPC, IN2P3-CNRS et Université de Caen, 14032 Caen, France \\ JINR, 141980 Dubna Russia \\ CFR, CNRS, 91190 Gif sur Yvette, France \\ ITEP-Moscow, Russia \\ INEEL, Idaho Falls, ID 83415, U.S.A. \\ JYVÄSKYLÄ University, 40351, Jyväskylä, Finland \\ LAL, IN2P3-CNRS et Université Paris-Sud, 91898 Orsay, France* \\ MHC, South Hadley, Massachusetts 01075, U.S.A. \\ IReS, IN2P3-CNRS et Université Louis Pasteur, 6703 Strasbourg, France \\ CTU FNSPE, Prague, 11519 Czech Republic \\ Charles University, Prague, Czech Republic \\ Saga University, Saga 840-8502, Japan \\ UCL, Gower Street London, WC 1 E $6 B T-U K$ \\ NOON03 Conference Kanazawa, Japan
}

Presented by

\title{
*S. Jullian
}

The NEMO collaboration is looking to measure neutrinoless double beta decay. The search for the effective neutrino mass will approach a lower limit of $0.1 \mathrm{eV}$. The NEMO 3 detector is now operating in the Frejus Underground Laboratory. The fundamental design of the detector is reviewed and the performances detailed. Finally, a summary of the data collected in the first runs which involve energy and time calibration and study of the background are presented.

\section{Introduction}

The recent discovery of neutrino oscillations is proof that the neutrino is a massive particle. However, the oscillation experiments are only sensitive to the difference in the square of the masses of two eigenstates of the neutrino. One method for directly measuring the absolute mass scale of neutrinos is through the 
careful investigation of the end-point energy of single beta decay. Another method could be through neutrinoless double beta decay $(\beta \beta(0 v))$ which is the mission of the NEMO 3 detector. The $\beta \beta(0 v)$ process is the decay of an $(A, Z)$ nucleus to an $(\mathrm{A}, \mathrm{Z}+2)$ nucleus by the simultaneous emission of two electrons but without the emission of neutrinos. The non-conservation of lepton number is a signature of physics beyond the Standard Model. An observation of $\beta \beta(0 v)$ would be one method of seeing this new physics and the measured half-life yields information on the effective neutrino mass.

In 1989, the NEMO (Neutrinoless Experiments with Molybdenum) collaboration started a research and development program to build a detector which would be able to study the effective neutrino mass down to about $0.1 \mathrm{eV}$ by looking for the $\beta \beta(0 v)$ decay process. The NEMO 3 detector [1] is now operating in the Frejus Underground Laboratory at a depth of 4800 m.w.e..

\section{The NEMO 3 detector}

\subsection{Description}

The detector is cylindrical in design and divided into 20 equal sectors (Fig. 1). A thin $\left(40-60 \mathrm{mg} / \mathrm{cm}^{2}\right)$ cylindrical source foil of $\beta \beta$ emitters has been constructed from either a metal film or powder bound by an organic glue to mylar strips. The detector houses up to $10 \mathrm{~kg}$ of these isotopes.

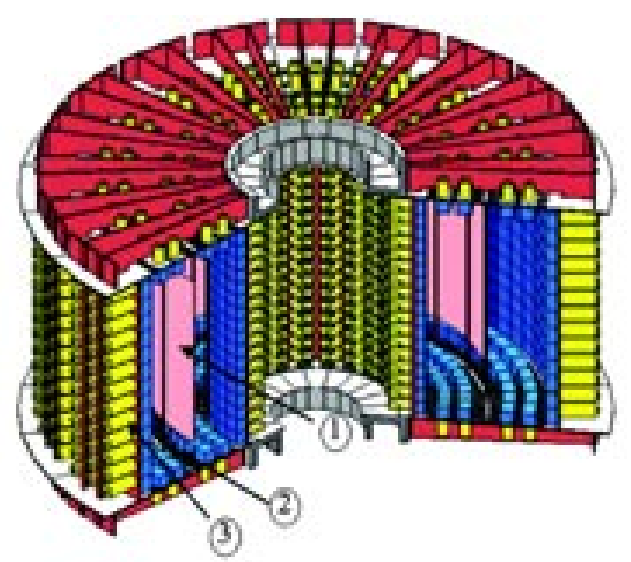

Figure 1: Schematic of the NEMO 3 detector: 1) Source foil (up to $10 \mathrm{~kg}$ ), 2) tracking volume with 6180 drift Geiger cells 3) Calorimeter of 1940 plastic scintillators coupled to low activity photomultipliers.

The double beta decay source hangs between two concentric cylindrical tracking volumes consisting of 6180 of open octagonal drift cells operating in 
geiger mode. These cells run vertically and are staged in a 4, 2, and 3 row pattern to optimize track reconstruction. The design of the drift cells calls for $50 \mu \mathrm{m}$ anode and cathode wires to prevent rapid aging. The tracking volume is filled with a mixture of $96 \%$ helium and $4 \%$ ethanol which operates at a pressure of $7 \mathrm{mbar}$ above the local atmospheric conditions. The typical length of a track is $1 \mathrm{~m}$ with the radial and longitudinal resolution in each geiger cell being $0.2 \mathrm{~mm}(1 \sigma)$ and $0.8 \mathrm{~cm}(1 \sigma)$, respectively. Consequently, the precision of the position of the emission vertex of the two electrons is 0.6 and $1 \mathrm{~cm}$ after track reconstruction in the transverse and longitudinal directions, respectively.

The external walls of these tracking volumes form a calorimeter made of blocks of plastic scintillator coupled to low radioactivity 3" and 5" Hammamatsu PMTs. The energy resolution depends on the scintillator shape and the associated PMT which range from $11 \%$ to $14.5 \%$ (FWHM) for $1 \mathrm{MeV}$ electrons. The time resolution is $250 \mathrm{ps}(1 \sigma)$ at $1 \mathrm{MeV}$. A laser calibration system permits daily checks on the stability of the energy and time calibration parameters. The detector contains 6180 drift cells and 1940 scintillators.

For charge recognition a solenoid surrounds the detector and produces a field of 30 Gauss to identify and reject pair production events. Finally, external shielding in the form of $20 \mathrm{~cm}$ of low activity iron reduces the gamma ray flux and then $30 \mathrm{~cm}$ of borated water suppresses the flux of neutrons.

The NEMO 3 detector's total mass is approximately 36 tons. All the materials used in the detector have been selected for their high radiopurity by $\gamma$ ray spectroscopy via Germanium detectors.

\subsection{NEMO 3's Self Study of Backgrounds}

The combination of a tracking volume, calorimeter, and magnetic field allow NEMO 3 to identify electrons, positrons, $\gamma$-rays and delayed- $\alpha$ particles. Thus, the detector can measure the internal contamination of the source by the er, ery or e $\alpha$ channels as well as reject the external background via additional cuts discussed later $[4,5]$.

An electron (or position) track in the detector corresponds to a curved trail of activitated drift cells with at least one end of the track ending in a scintillator which has registered energy. A $\gamma$-ray corresponds to a scintillator being triggered without an associated track. Finally, an alpha particle is a short track without curvature and possibly delayed by up to $1 \mathrm{~ms}$.

In Figures 2, 3, 4 and 5 one can see events characteristic of some backgrounds.

Figure 2 shows pair production in the source foil. In Figure 3 one can see the decay of some "internal contamination" which yields an electron followed by a delayed alpha. Figure 4 shows an event which is known to come from an "external" source. Finally, Figure 5 shows an event with one single $\mathrm{e}^{-}$and $3 \gamma$ rays. The most sensitive channel [2] to see the external background caused 


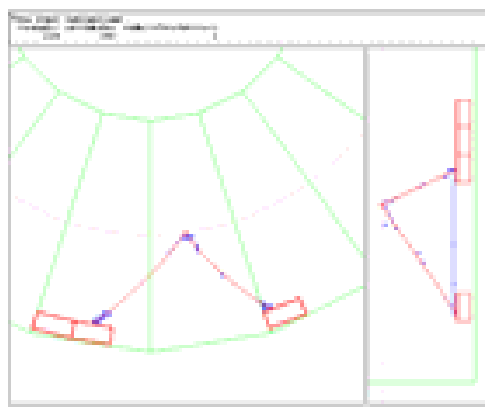

Figure 2: $\mathrm{e}^{-} \mathrm{e}^{+}$pair production

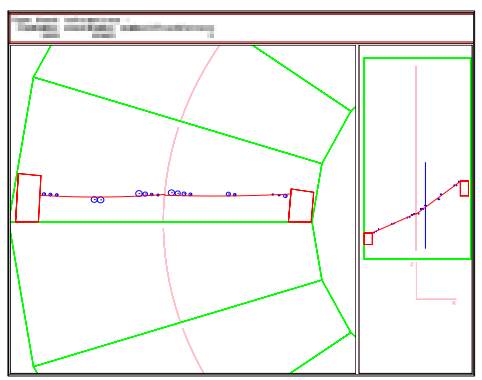

Figure 4: one crossing electron

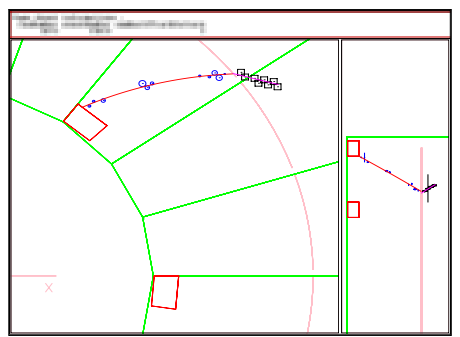

Figure 3: single $\mathrm{e}^{-}$and delayed $\alpha$

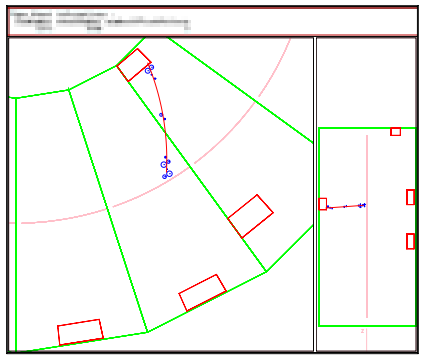

Figure 5: single $\mathrm{e}^{-}$and $3 \gamma$ rays

by neutrons interacting with the detector is the one-crossing-electron channel corresponding to Compton electrons created in a scintillator and then crossing the detector. This kind of event is distinguished from the two electron events emitted from the source by time-of-flight measurements.

\subsection{The Double Beta Sources in NEMO 3}

\subsubsection{Isotopes and Radiopurity}

Several sources were placed in the detector to study $\beta \beta(0 v)$ decay but also to measure other processes such as $\beta \beta(2 v)$ decay, the Majoron decay mode and the external backgrounds. Table 1 summarizes the isotopes currently housed in NEMO 3 with their total mass and decay mode of interest. 


\begin{tabular}{|c|c|c|c|c|c|}
\hline Isotope & Enrichment & Decay of Interest & \multicolumn{2}{|c|}{ Mass } & \multicolumn{2}{|c|}{$\begin{array}{c}\text { Se Spectrometer Measurements } \\
\text { of Isotope }(\mathrm{g})\end{array}$} & $\begin{array}{c}214 \\
\mathrm{Bi}(\mathrm{mBq} / \mathrm{kg})\end{array}$ & $\begin{array}{c}208 \\
\mathrm{Tl}(\mathrm{mBq} / \mathrm{kg})\end{array}$ \\
\hline${ }^{100} \mathrm{Mo}$ & $97 \%$ & $\beta \beta(0 v)$ & 6914 & $<0.3$ & $<0.17$ \\
\hline${ }^{82} \mathrm{Se}$ & $97 \%$ & $\beta \beta(0 v)$ & 932 & $1.2 \pm 0.5$ & $0.4 \pm 0.1$ \\
\hline${ }^{130} \mathrm{Te}$ & $89 \%$ & $\beta \beta(2 v)$ & 454 & $<0.7$ & $<0.5$ \\
\hline${ }^{116} \mathrm{Cd}$ & $93 \%$ & $\beta \beta(2 v)$ & 405 & $<2$ & $<0.8$ \\
\hline${ }^{150} \mathrm{Nd}$ & $91 \%$ & $\beta \beta(2 v)$ & 36.6 & $<3.3$ & $10 \pm 2$ \\
\hline${ }^{96} \mathrm{Zr}$ & $57 \%$ & $\beta \beta(2 v)$ & 9.4 & $<17$ & $<10$ \\
\hline${ }^{48} \mathrm{Ca}$ & $73 \%$ & $\beta \beta(2 v)$ & 7.0 & $<4$ & $<2$ \\
\hline${ }^{10 \mathrm{Ca}} \mathrm{Te}$ & & External $\gamma \mathrm{bkg}$ & 207 & $<0.17$ & $<0.17$ \\
\hline $\mathrm{Cu}$ & & External $\gamma \mathrm{bkg}$ & 621 & $<0.12$ & $<0.03$ \\
\hline
\end{tabular}

Table 1: List of enriched isotopes placed in the NEMO 3 detector with limits on their activity.

However, note that with the ${ }^{100} \mathrm{Mo},{ }^{82} \mathrm{Se}$ and ${ }^{116} \mathrm{Cd}$ isotopes one can not only search for $\beta \beta(0 v)$ decay, but also $\beta \beta(2 v)$ decay to the ground and excited states, and the Majoron emission decay $\beta \beta(\chi)$ modes.

For ${ }^{100} \mathrm{Mo}, 0.1$ million $\beta \beta(2 v)$ events per year will be recorded giving high statistics for the angular distribution between the two emitted electrons and the single electron energy spectrum.

The other enriched isotopes $\left({ }^{130} \mathrm{Te},{ }^{150} \mathrm{Nd},{ }^{96} \mathrm{Zr}\right.$ and $\left.{ }^{48} \mathrm{Ca}\right)$ were installed to measure the $\beta \beta(2 v)$ half-life for comparison with the predictions of their respective nuclear matrix element calculations. The natural tellurium and copper are very pure, so that the events associated with these sources, in the $3 \mathrm{MeV}$ region, are induced by the external $\gamma$-ray flux and as such provide limits on this flux.

Another interest in the varied sources is that for long range planning it is useful to measure the contamination after enrichment and study purification and source foil production for future improvements in NEMO 3.

\subsubsection{Purification}

The enrichment process failed to yield the desired levels of purity for the ${ }^{100}$ Mo so purification techniques were developed. These techniques started with the enriched material which was a fine grey powder. In the end two techniques were developed for purification and foil production.

The first technique is a physical process that was developed at ITEP. The powder is melted by an electron beam and a crystal of pure material is drawn into a long narrow cylinder. The crystal cylinders are then cut to a fiducial length and foils are obtained by rolling the crystal in a vacuum between very pure steel rollers. This process had a total yield of $2.479 \mathrm{~kg}$ of ${ }^{100} \mathrm{Mo}$ for the experiment.

The second process was chemical in nature [3]. A flow chart of the chemical purification technique is shown below. The chemistry proceeds as 
follows. The majority of the process was carried out in a class 100 clean room at INEEL. The laboratory ware is comprised of cleaned quartz, Teflon and one piece plastic disposible filter units. Quartz distilled nitric acid and ultra-purified (18 M 2 ) water were used to dissolve the Mo. Research grade $\mathrm{He}$ and $\mathrm{H}_{2}$ gases were used during the Mo reduction cycle.

The expected purification factor is more than 100 as indicated by a study made with a sample of natural molybdenum. This sample originally had an activity of $28 \mathrm{mBq} / \mathrm{kg}$ before purification and less than $0.3 \mathrm{mBq} / \mathrm{kg}$ after the process. If one applies this factor to the ${ }^{100}$ Mo used in NEMO 3, which had a typical activity of $1.3 \mathrm{mBq} / \mathrm{kg}$ you expect the final product to be at a level of $0.013 \mathrm{mBq} / \mathrm{kg}$ or better. This process was applied to the $4.260 \mathrm{~kg}$ of chemically purified ${ }^{100}$ Mo used in NEMO 3. This level of contamination will be measured easily with the NEMO 3 detector through the $e \gamma \gamma$ and $e \gamma \gamma \gamma$ channels. NEMO 3 is sensitive to $0.002 \mathrm{mBq} / \mathrm{kg}$ after one year of exposure.

\section{Performances of the tracking detector}

\subsection{Generation of high-energy electrons crossing}

An Am/Be neutron source, situated on the bottom of the detector, emits fast neutrons, thermalised in the plastic of the scintillators ; then, a radiative capture of these thermalised neutrons, in the copper present inside the detector produces a $\gamma$ whose energy can go up to $8 \mathrm{MeV}$. The Compton electron created by this $\gamma$ can cross all the detector, from one wll of scintillators the opposited one. Using crossing electron with an energy higher than $4.5 \mathrm{MeV}$, we can study properly the tracking reconstruction, since the effects of multiple scattering become negligible.

From these crossing electrons, we could determine the law between the drift time and the drift distance, inside a Geiger cell, necessary for the transversal reconstruction of the tracks : the drift time is, excepted for very short $(\leq 200 \mathrm{~ns})$ or very long ( $\geq 1500 \mathrm{~ns}$ ) drift times, roughly proportional to the racine square of the drift distance, which is logical since the electrostatique field inside a Geiger cell is inversement porportionnal to the drift distance.

Looking at the distribution of the residue, defined as the difference between the drift distance and the transversal reconstructed distance, inside a Geiger cell, we could détermine the average transversal and longitudinal resolutions for a drift cell, equal to $0.4 \mathrm{~mm}$ and $0.8 \mathrm{~cm}$, respectivement.

The charge recognition, ensured by the existence of the magnetic field, had to be checked ; therefore, we used the same sample of electron crossing events, and constrained the first part of the track - from the first wall of PM to the source foil - to be reconstructed as an electron. Therefore, the study of the second part of the track - from the source foil to the opposite wall - gives us the probability to confuse an electron and a positron, equal to $3 \%$ at $1 \mathrm{MeV}$. 


\subsubsection{Spatial resolution on the vertex}

In each sector of the detector, there is a copper tube at the radius of the foil, which runs vertically for the height of the detector; during energy calibration runs, each tube has inserted into it three ${ }^{207} B i$ sources of $5 \mathrm{nCi}$, for a total of sixty sources. As the position of these sources are very well-known, the study of the two conversion electrons $(0.5$ and $1 \mathrm{MeV})$ emitted by these sources drives us to the determination of the spatial resolution on the vertex of the tracks. In the 1electron channel, the transversal and longitudinal resolutions are equal to $0.2 \mathrm{~cm}$ and $0.8 \mathrm{~cm}$, respectively, at $1 \mathrm{MeV}$. In the 2-electron channel, useful compte tenu of the signal searched by NEMO 3, they are equal to $0.6 \mathrm{~cm}$ and $1.0 \mathrm{~cm}$, respectively.

\section{Radiopurity in ${ }^{208} \mathrm{Tl}$ of the sources of ${ }^{100} \mathrm{Mo}$}

\subsection{Principle}

Since a ${ }^{208} \mathrm{Tl}$ nucleus emits, in $100 \%$ of its desintegration, a $\gamma$-ray with the highest energy of the natural radioactivity $(2.6 \mathrm{MeV})$, close to the endpoint of the $\beta \beta$ process, situated around $3 \mathrm{MeV}$ for the nuclei studied in NEMO 3, this nucleus is the most dangerous background for $\beta \beta 0 v$ study. Therefore, the pollution in ${ }^{208} \mathrm{Tl}$ of the foils has to be very well known. The principle of the study lies on the exploitation of the e-n $\gamma(1 \leq n \leq 3)$ channels, with appropriated cuts on the energy of the different particles, but also on the time of flight of them (through a $\chi^{2}$ analysis), in order to select events emitted in the source foil.

The cuts applied on the total energy of the photon(s), on the energy of the electron, on the $\chi_{\text {intemal }}$ that characterizes temporally events emitted in the foil sources, the efficiencies related to the three channels, are shown in Table 2 . We can consider that the possible background contribution to this analysis, which consists in external or internal ${ }^{214} \mathrm{Bi}$ and external ${ }^{208} \mathrm{Tl}$, is negligible with these cuts.

\begin{tabular}{|lc|c|c|c|}
\hline $\begin{array}{c}\text { Channel } \\
\text { of the photons (MeV) }\end{array}$ & $\begin{array}{c}\text { Total energy } \\
\text { The electron }\end{array}$ & $\chi^{2}$ internal & Efficiency (\%) \\
\hline $\mathrm{e}^{-} \gamma$ & $\geq 2.3$ & $0.5 \leq \mathrm{E}_{\mathrm{e}_{-}} \leq 1.3$ & 6.7 & $0.294 \pm 0.005$ \\
\hline $\mathrm{e}^{-} 2 \gamma$ & $\geq 2.3$ & $0.5 \leq \mathrm{E}_{\mathrm{e}_{-}} \leq 1.3$ & 13.8 & $0.369 \pm 0.006$ \\
\hline $\mathrm{e}^{-} 3 \gamma$ & $\geq 2.6$ & $0.5 \leq \mathrm{E}_{\mathrm{e}_{-}} \leq 1.3$ & 16.3 & $0.114 \pm 0.003$ \\
\hline
\end{tabular}

Table 2: Characteristics of the differents channels studied for the determination of the ${ }^{208} \mathrm{Tl}$ pollution inside the ${ }^{100}$ Mo source: cuts applied and efficiency (with statistical error), determined through simulated events. 


\subsection{Preliminary results}

Using 900 hours of data, taken in unstable conditions, we get the upper limits on the ${ }^{208} \mathrm{Tl}$ pollution of the ${ }^{100} \mathrm{Mo}$ sources shown in Table 3 ; if we

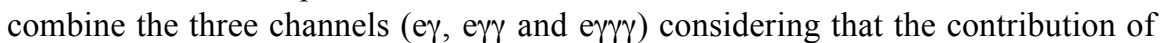
the other backgrounds (external or internal ${ }^{214} \mathrm{Bi}$ and external ${ }^{208} \mathrm{Tl}$ ) to the search for an internal ${ }^{208} \mathrm{Tl}$ signal is negligible, the upper limit on the ${ }^{208} \mathrm{Tl}$ activity of the ${ }^{100} \mathrm{Mo}$ source foils is equal to $68 \mu \mathrm{Bq} / \mathrm{kh}$, at $90 \%$ C.L.. This limit, already better than the one measured by $\gamma$-ray spectroscopy using HP Ge detectors $(\leq$ $110 \mu \mathrm{Bq} / \mathrm{kg}$ ) is not so far from the limit required by the NEMO 3 radiopurety criteria $(\leq 20 \mu \mathrm{Bq} / \mathrm{kg})$. We expect to reach this last limit within four months of data.

\begin{tabular}{|lc|}
\hline Channel & Activities (90\% C.L.) \\
\hline $\mathrm{e}^{-} \gamma$ & $\leq 85 \mu \mathrm{Bq} / \mathrm{kg}$ \\
\hline $\mathrm{e}^{-} 2 \gamma$ & $\leq 78 \mu \mathrm{Bq} / \mathrm{kg}$ \\
\hline $\mathrm{e}^{-} 3 \gamma$ & $\leq 86 \mu \mathrm{Bq} / \mathrm{kg}$ \\
\hline
\end{tabular}

Table 3: Upper limits (90\% C.L.) on the reconstructed activities in ${ }^{208} \mathrm{Tl}$ of the ${ }^{100} \mathrm{Mo}$ source foils, using a sample of events representing 900 hours of acquisition.

\section{Double beta analysis}

Using the same sample of events, representing 900 hours of first test data, we could perform preliminary analysis of $\beta \beta 2 v$ and $\beta \beta 0 v$ signals. In this paper, this analysis is presented for ${ }^{100} \mathrm{Mo}$ only. Therefore, the events selected have to be emitted on the same point of a ${ }^{100}$ Mo foil: geometrical cuts on the vertex of the electrons emitted and temporal cuts (based on a $\chi^{2}$ analysis) devoted to the selection of electrons emitted inside the source foils, are applied. Figure 6 shows a typical $\mathrm{e}^{-} \mathrm{e}^{-}$event.

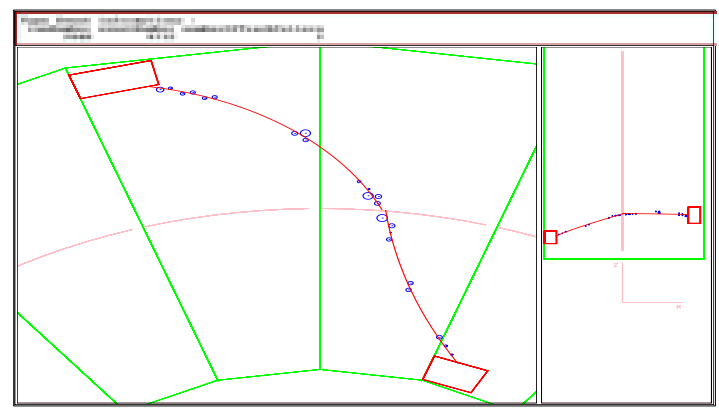

Figure 6: an $\mathrm{e}^{-} \mathrm{e}^{-}$event back to back 
Using the sample of events described below, representing 15107 events, we performed a preliminary analysis of $\beta \beta 2 v$ decay. The spectrum of the sum of the kinetic energies of both electrons is shown in Fig. 7, and the angular distribution is shown in Fig. 8, there is a good agreement between data and Monte-Carlo. The half-life deduced from these data is equal to $9 . \pm 0.08$ (stat.) \pm 1.3 (syst.) $10^{18} \mathrm{y}$ : it is already of the same order of magnitude than the results give by NEMO2. The radio signal over background is greater then 100 .

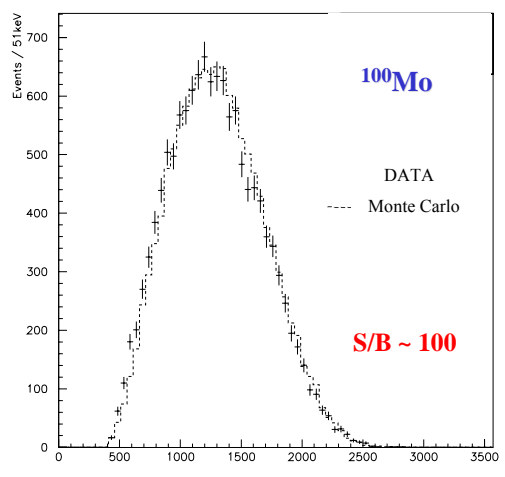

Figure 7: $\mathrm{e}^{-} \mathrm{e}^{-}$energy distribution

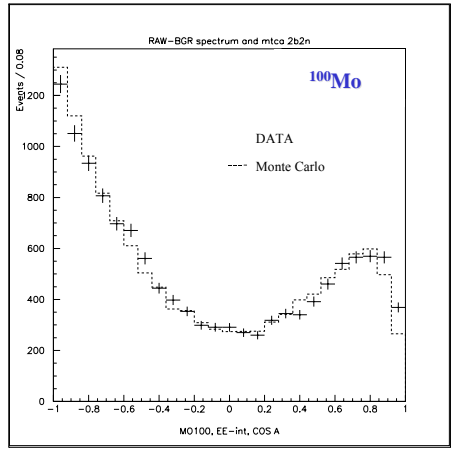

Figure 8: $\mathrm{e}^{-} \mathrm{e}^{-}$angular distribution

\section{Conclusion}

With the first test runs taken by the NEMO 3 experiment, we have measured the main characteristics of the detector, in good agreement with what we expected: the energy and time calibration have been performed and the performances of the tracking detector have been determined. Moreover, the preliminary analysis of the ${ }^{208} \mathrm{Tl}$ pollution of the ${ }^{100}$ Mo source foils gave an upper limit on this pollution, equal to $68 \mu \mathrm{Bq} / \mathrm{kg}$, at $90 \%$ C.L., the limit required by NEMO 3 should be reached within four months of data. Finally, the $\beta \beta 2 v$ ananlysis show a good agreement between Monte Carlo and data, and will be applied to the data taken by the full detector, in stable conditions.

\section{References}

[1] NEMO Collaboration, NEMO 3 Proposal, LAL 94-29 (1994)

[2] C. Marquet et al, Nucl. Instrum. and Methods A 457, 487 (2000)

[3] R. Arnold et al, Nucl. Instrum. and Methods A 474, 93 (2001)

[4] H. Ohsumi et al, Nucl. Instrum. and Methods A 482, 832 (2002)

[5] R. Arnold et al, Nucl. Instrum. and Methods A 457, 487 (2000) 